\title{
Groenewold-von Neumann product via Segal-Bargmann transform
}

\author{
JOHN B. MORENO* \\ Universidad del Atlántico, Programa de Matemáticas, Barranquilla, Colombia.
}

\begin{abstract}
Using standard techniques from geometric quantization, we rederive the product of functions on $\mathbb{R}^{2}$ which was first introduced by von Neumann and later reintroduced by Groenewold and which is the integral version of the Moyal product. More specifically, by pairing the diagonal real polarization on the pair groupoid $\mathbb{R}^{2} \times \overline{\mathbb{R}}^{2}$ with its standard holomorphic polarization, we obtain the well-known Segal-Bargmann transform in a rotated and scaled (and half-conjugated) form. Together with a convolution of functions in the Segal-Bargmann space, which is a natural deformation of the usual convolution of functions on the pair groupoid, this defines the Groenewold-von Neumann product on $L^{2}\left(\mathbb{R}^{2}\right)$.
\end{abstract}

Keywords: Geometric quantization, star product, Segal-Bargmann transform, Fock spaces.

MSC2010: 53D50, 53D55, 30H20.

\section{Producto de Groenewold-von Neumann mediante una transformada de Segal-Bargmann}

Resumen. Usando técnicas de cuantización geométrica, obtenemos el producto de funciones en $\mathbb{R}^{2}$, primeramente introducido por von Neumann y posteriormente reintroducido por Groenewold, el cual es la versión integral del producto de Moyal-Weyl. De forma más específica, por el empareamiento de polarizaciones reales en el par grupoide $\mathbb{R}^{2} \times \overline{\mathbb{R}}^{2}$ con sus polarizaciones holomorfas estándares, obtenemos una transformada de Segal-Bargamann deformada (por rotación y traslación). Junto con una convolución de funciones en el espacio de Segal-Bargmann, la cual es una deformación natural de la convolución de funciones en el par grupoide, se obtiene el producto de Groenewold-von Neumann en $L^{2}\left(\mathbb{R}^{2}\right)$.

Palabras clave: Cuantización geométrica, producto estrella, transformada de Segal-Bargmann, espacios Fock.

\footnotetext{
*E-mail: johnmoreno@mail.uniatlantico.edu.co

Received: 25 July 2015, Accepted: 02 September 2015.

To cite this article: J.B. Moreno, Groenewold-von Neumann product via Segal-Bargmann transform, Rev Integr. Temas Mat. 33 (2015), No. 2, 135-144.
} 


\section{Introduction}

Let $M$ be a symplectic symmetric space, $T M$ its tangent bundle, and let $M \times \bar{M}$ be the symplectic pair groupoid. Since $M$ is a symplectic symmetric space, the pushforward of the vertical fibration of $T M$ under the map

$$
\Phi: T M \rightarrow M \times M, \quad(m, v) \mapsto\left(\exp _{m}(-v), \exp _{m}(v)\right)
$$

determines a foliation $\mathcal{F}_{V}$ on $M \times \bar{M}$ which, if regular, defines a real polarization on the symplectic pair groupoid (cf. [4],[13], for instance). The regularity condition fails if $M$ is compact, but it is satisfied if $M$ is noncompact with no compact factors. This short paper considers only the simplest possible case: $M=\mathbb{R}^{2 n}$ (actually we here fix $n=1$ to make matters simpler without any significant loss of generality).

Now, the integral version of the Weyl-Moyal product of functions on $\mathbb{R}^{2 n}$, also known as the Groenewold-von Neumann product, has been obtained and re-obtained in various ways since the original work of von Neumann [11]. But in [5], Gracia-Bondia and Varilly re-derived this product via geometric quantization, using the pairing of two nontransversal real polarizations on the pair groupoid $\mathbb{R}^{2 n} \times \overline{\mathbb{R}^{2 n}}$, one being the polarization $\mathcal{F}_{V}$ described above (more recently, the polarization $\mathcal{F}_{V}$ has been used together with an "averaging procedure" to re-derive this product via geometric quantization [13]). Here we will once again re-derive this product, again via geometric quantization and again using pairing of polarizations, but now pairing the real polarization $\mathcal{F}_{V}$ to a transversal holomorphic polarization on $\mathbb{R}^{2 n} \times \overline{\mathbb{R}^{2 n}}$.

Although our derivation presented below could be considered as a simple exercise in geometric quantization, we have not yet found it explicitly done in detail in the literature. In fact, the main idea for this derivation is already found in the aforementioned paper by Gracia-Bondía and Várilly ([5], Section VI), but their treatment there is somewhat sketchy and incomplete and uses references to previous papers by Daubechies and Grossmann ([2],[3]). On the other hand, appropriate generalizations of this technique to other noncompact hermitian symmetric spaces can in principle be helpful. For instance, if $M=\mathbb{H}^{2}$ is the hyperbolic plane, it is not possible to find another real polarization on the symplectic pair groupoid whose degree of transversality to $\mathcal{F}_{V}$ is everywhere constant, but in contrast, the standard holomorphic polarizations on $\mathbb{H}^{2} \times \overline{\mathbb{H}}^{2}$ are everywhere transversal to $\mathcal{F}_{V}$. This fact shall be thoroughly explored in subsequent papers and constitutes the main motivation for us to working out this technique in detail for the case of $\mathbb{R}^{2}$ in this note.

As we shall see below in detail, the geometric quantization pairing of $\mathcal{F}_{V}$ and a standard holomorphic polarization on $\mathbb{R}^{2} \times \overline{\mathbb{R}^{2}}$ defines a Segal-Bargmann transform from functions on $\mathbb{R}^{2}$ to holomorphic functions on $\mathbb{C} \times \overline{\mathbb{C}}$, which is isometric to the standard SegalBargmann transform. This latter, originally introduced by V. Bargmann [1], has many applications in quantum optics as well as in signal processing and harmonic analysis on phase space [6], and is usually defined by

$$
\mathfrak{B}[f](z)=\frac{e^{-\frac{1}{2} z^{2}}}{\pi^{n / 4}} \int_{\mathbb{R}^{n}} f(x) e^{-\frac{1}{2} x^{2}+\sqrt{2} x z} d x, z \in \mathbb{C}^{n},
$$

where $z^{2}=z_{1}^{2}+z_{2}^{2}+\cdots z_{n}^{2}$, similarly for $x^{2}$, with $x z=x_{1} z_{1}+x_{2} z_{2}+\cdots x_{n} z_{n}$. 
Then, $\mathfrak{B}$ maps isometrically the space $L^{2}\left(\mathbb{R}^{n}, d x\right)$ of square integrable functions on $\mathbb{R}^{n}$ onto the Segal-Bargmann space $\mathcal{H} L^{2}\left(\mathbb{C}^{n}\right)$ of holomorphic functions on $\mathbb{C}^{n}$ which are $e^{-|z|^{2}} d \mu(z)$-square integrable, $d \mu(z)$ being the Lebesgue measure on $\mathbb{C}^{n}$.

It is well known that geometric quantization can be used to construct the Segal-Bargmann space for $\mathbb{C}^{n}$ and its associated Segal-Bargmann transform (cf. e.g. [7],[12],[14]), and the generalized Segal-Bargmann transform for Lie Groups of compact type can also be developed using geometric quantization (cf. [8],[9]).

In this short note, again via geometric quantization, we shall obtain the 2-d SegalBargmann transform in a "rotated and scaled" form:

$$
\widetilde{\mathfrak{B}}_{\hbar}[f]\left(w_{1}, w_{2}\right)=C_{\hbar} e^{-\frac{1}{2 \hbar} w_{1} w_{2}} \int_{\mathbb{R}^{2}} f\left(y_{1}, y_{2}\right) e^{-\frac{1}{\hbar}\left(y_{1}^{2}+y_{2}^{2}\right)} e^{\frac{1}{\hbar}\left[y_{1}\left(w_{1}+w_{2}\right)-i y_{2}\left(w_{1}-w_{2}\right)\right]} d y_{1} d y_{2},
$$

where $w=\left(w_{1}, w_{2}\right) \in \mathbb{C}^{2}, y=\left(y_{1}, y_{2}\right) \in \mathbb{R}^{2}$, and Planck's constant $\hbar \in \mathbb{R}^{+}$can also be considered as a free positive parameter whenever this is convenient.

Now, (3) takes the form (2) under the linear changes of variables:

$$
\begin{aligned}
\eta_{\hbar}: \mathbb{C}^{2} \rightarrow \mathbb{C}^{2}, \quad w & \mapsto z=\left(z_{1}=\frac{1}{2 \sqrt{\hbar}}\left(w_{1}+w_{2}\right), z_{2}=\frac{i}{2 \sqrt{\hbar}}\left(w_{2}-w_{1}\right)\right), \\
\zeta_{\hbar} & : \mathbb{R}^{2} \rightarrow \mathbb{R}^{2}, \quad y \mapsto x=y \sqrt{2 / \hbar},
\end{aligned}
$$

so that

$$
\widetilde{\mathfrak{B}}_{\hbar}[f]=\mathfrak{B}\left[f \circ \zeta_{\hbar}^{-1}\right] \circ \eta_{\hbar}
$$

for an appropriate choice of constant $C_{\hbar}$, and therefore both forms of the Segal-Bargmann transform, albeit appearing different, are fully equivalent.

That is, observing that under the linear change $\eta_{\hbar}$ given by (4) we have that

$$
e^{-|z|^{2}} d \mu(z)=\frac{1}{4 \hbar} e^{-|w|^{2} / 2 \hbar} d \mu(w),
$$

then the map $\mathfrak{B} \mapsto \widetilde{\mathfrak{B}}_{\hbar}$ defined by (6) is, modulo an overall constant factor, an isometry from $\mathcal{H} L^{2}\left(\mathbb{C}^{2}\right)$ to $\mathcal{H} L_{2 \hbar}^{2}\left(\mathbb{C}^{2}\right)$, where $\mathcal{H} L_{t}^{2}\left(\mathbb{C}^{2}\right), t>0$, is the space of holomorphic functions on $\mathbb{C}^{2}$ which are $e^{-|z|^{2} / t} d \mu(z)$-square integrable. It follows that $\widetilde{\mathfrak{B}}_{\hbar}$ given by (3) is an isometry from $L^{2}\left(\mathbb{R}^{2}, d y\right)$ to $\mathcal{H} L_{2 \hbar}^{2}\left(\mathbb{C}^{2}\right)$, and conjugating the second variable it produces the transform from $L^{2}\left(\mathbb{R}^{2}, d y\right)$ to $\mathcal{H} L_{2 \hbar}^{2}(\mathbb{C} \times \overline{\mathbb{C}})$ (cf. Theorem 2.3 , which is also referred to as the Daubechies-Grossmann transform [2],[3]).

Thus, in Section 2 of this short paper we present our detailed derivation of this transform (cf. (19)-(24)), which immediately generalizes to all even dimensional cases. Then, in Section 3, combining this transform with a natural deformation of the usual convolution of functions on the pair groupoid, we obtain the integral formulation of the Moyal-Weyl product, i.e., the Groenewold-von Neumann product of (complex) functions on the real plane, which is given by (cf. e.g. [13]):

$\left[g_{1} *_{\hbar} g_{2}\right](x, y)=\int_{\mathbb{R}^{2} \times \mathbb{R}^{2}} g_{1}\left(x_{1}, y_{1}\right) g_{2}\left(x_{2}, y_{2}\right) e^{\frac{i}{\hbar}\left(x_{1} y_{2}-x_{2} y_{1}+x y_{1}-x_{1} y+x_{2} y-x y_{2}\right)} d x_{1} d y_{1} d x_{2} d y_{2}$.

Vol. 33, No. 2, 2015] 


\section{A Segal-Bargmann transform via geometric quantization}

Let $\mathbb{R}^{2}$ be the plane with coordinates $(x, y)$ and canonical symplectic form $\omega=d x \wedge d y$. In this case, the map

$$
\Phi: T \mathbb{R}^{2} \longrightarrow \mathbb{R}^{2} \times \mathbb{R}^{2}, \quad(m, v) \mapsto\left(\exp _{m}(-v), \exp _{m}(v)\right)
$$

of (1), is given explicitly by

$$
\Phi\left(x, y ; v_{x}, v_{y}\right)=\left(x-v_{x}, y-v_{y} ; x+v_{x}, y+v_{y}\right)=\left(x_{-}, y_{-} ; x_{+}, y_{+}\right) .
$$

Denote by $\mathbb{R}^{2} \times \overline{\mathbb{R}}^{2}$ the symplectic manifold with symplectic form given by $\Omega=\omega-\omega$. If $\mathbb{R}^{2} \times \overline{\mathbb{R}}^{2}$ has coordinates $\left(x_{-}, y_{-} ; x_{+}, y_{+}\right)$as above, then $\Omega=d x_{-} \wedge d y_{-}-d x_{+} \wedge d y_{+}$, and since $\Phi$ is a diffeomorphism with inverse $\Phi^{-1}$ given by

$$
x=\frac{x_{-}+x_{+}}{2}, \quad y=\frac{y_{-}+y_{+}}{2} ; \quad v_{x}=\frac{x_{+}-x_{-}}{2}, \quad v_{y}=\frac{y_{+}-y_{-}}{2},
$$

the pull-back symplectic form $\Phi^{*}(\Omega)=\Pi$ on $T \mathbb{R}^{2}$ is given by

$$
\Pi=2\left(d v_{y} \wedge d x-d v_{x} \wedge d y\right) .
$$

On the other hand, taking

$$
z_{-}=x_{-}+i y_{-}, \quad z_{+}=x_{+}+i y_{+}
$$

as holomorphic coordinates in $\mathbb{R}^{2} \times \mathbb{R}^{2} \simeq \mathbb{C} \times \mathbb{C}$, then

$$
\Omega=\frac{i}{2} d z_{-} \wedge d \bar{z}_{-}-\frac{i}{2} d z_{+} \wedge d \bar{z}_{+} .
$$

Consider the following respective polarizations on $T \mathbb{R}^{2}$ and $\mathbb{R}^{2} \times \overline{\mathbb{R}}^{2}$ :

$$
\widetilde{P}=\left\langle\partial v_{x}, \partial v_{y}\right\rangle \text { and } F=\left\langle\partial \bar{z}_{-}, \partial z_{+}\right\rangle .
$$

From (11), the symplectic potential adapted to the polarization $\widetilde{P}$ is given by

$$
\Theta_{\widetilde{P}}=2\left(v_{y} d x-v_{x} d y\right),
$$

while from (13) the symplectic potential adapted to the polarization $F$ is given by

$$
\Theta_{F}=-\frac{i}{2} \bar{z}_{-} d z_{-}-\frac{i}{2} z_{+} d \bar{z}_{+} .
$$

For $\widetilde{F}=\left(\Phi^{-1}\right)_{*} F$, we have from (9)-(10) that

$\Theta_{\widetilde{F}}=-\left[\left(v_{x} d y+x d v_{y}-y d v_{x}-v_{y} d x\right)+\frac{i}{4} d\left(\left(x-v_{x}\right)^{2}+\left(y-v_{y}\right)^{2}+\left(x+v_{x}\right)^{2}+\left(y+v_{y}\right)^{2}\right)\right]$.

Therefore,

$$
\Theta_{\widetilde{P}}-\Theta_{\widetilde{F}}=d \widetilde{\Psi}
$$


where

$$
\widetilde{\Psi}\left(x, y, v_{x}, v_{y}\right)=\left(x v_{y}-y v_{x}\right)+\frac{i}{4}\left[\left(x-v_{x}\right)^{2}+\left(y-v_{y}\right)^{2}+\left(x+v_{x}\right)^{2}+\left(y+v_{y}\right)^{2}\right],
$$

which in terms of the holomorphic coordinates on $\mathbb{R}^{2} \times \mathbb{R}^{2}$ can be written as

$$
\widetilde{\Psi} \circ \Phi^{-1}=\Psi\left(z_{-}, \bar{z}_{-}, z_{+}, \bar{z}_{+}\right)=\frac{i}{4}\left(\bar{z}_{+} z_{-}-\bar{z}_{-} z_{+}+z_{-} \bar{z}_{-}+z_{+} \bar{z}_{+}\right),
$$

with explicit expressions in holomorphic coordinates for the map

$$
\Phi^{-1}\left(z_{-}, \bar{z}_{-} ; z_{+}, \bar{z}_{+}\right)=\left(x, y ; v_{x}, v_{y}\right)
$$

obtained by combining (10) and (12).

Now, recall that a connection on a hermitian line bundle $L$ associated to the pre-quantum principal $S^{1}$-bundle over a symplectic manifold $M$ is given locally by

$$
\nabla_{X}=X-(i / \hbar) \Theta(X), \quad X \in \mathfrak{X}(M),
$$

where $\Theta$ is a symplectic potential. Then, consider the polarized section $s_{0}$ of $L$ over $\mathbb{R}^{2} \times \overline{\mathbb{R}}^{2}$ adapted to the symplectic potential $\Theta_{F}$ and its pull-back $\widetilde{s}_{0}$ adapted to $\Theta_{\widetilde{F}}$, as well as the polarized section $\widetilde{t}_{0}$ of $\tilde{L}$ over $T \mathbb{R}^{2}$ adapted to the symplectic potential $\Theta_{\widetilde{P}}$ and its push-forward $t_{0}$ adapted to $\Theta_{P}$, where $P=\Phi_{*} \widetilde{P}$, satisfying

$$
\begin{aligned}
\nabla_{\widetilde{X}} \widetilde{t}_{0}=-(i / \hbar) \Theta_{\widetilde{P}} \widetilde{t}_{0} ; & \left(\widetilde{t_{0}}, \widetilde{t}_{0}\right)=1, \\
\nabla_{\widetilde{X}} \widetilde{s}_{0}=-(i / \hbar) \Theta_{\widetilde{F}} \widetilde{s}_{0} ; & \left(\widetilde{s}_{0}, \widetilde{s_{0}}\right)=1,
\end{aligned}
$$

where $(\cdot, \cdot)$ is the hermitian product of the line bundle $\tilde{L}$ and $\tilde{X} \in \mathfrak{X}\left(T \mathbb{R}^{2}\right)$, with similar expressions for $t_{0}$ and $s_{0}$ in terms of $P, F,(\cdot, \cdot)$ on $L$, and $X \in \mathfrak{X}\left(\mathbb{R}^{2} \times \overline{\mathbb{R}}^{2}\right)$.

The polarized sections $\tilde{t} \in \Gamma_{\widetilde{P}} \tilde{L}$ are given by $\widetilde{t}=\bar{g} \widetilde{t}_{0}$, with $\bar{g} \in \mathcal{C}_{\mathbb{C}}^{\infty}\left(T \mathbb{R}^{2}\right)$ satisfying $X \bar{g}=0$, for $X \in \mathfrak{X}\left(T \mathbb{R}^{2}, \widetilde{P}\right)$; thus, it follows that $\bar{g}$ depends only on the variables $(x, y) \in \mathbb{R}^{2}$ seen as the zero section of $T \mathbb{R}^{2}$. Similarly, the polarized sections $s \in \Gamma_{F} L$ are of the form $s=f s_{0}$, where $\frac{\partial f}{\partial \bar{z}_{-}}=\frac{\partial f}{\partial z_{+}}=0$, that is, $f=f\left(z_{-}, \bar{z}_{+}\right)$is holomorphic.

Furthermore, as the pre-quantum line bundle is a linear bundle, we have that $\widetilde{s}_{0}=\tilde{\phi} \widetilde{t}_{0}$ for a nonvanishing function $\tilde{\phi} \in \mathcal{C}_{\mathbb{C}}^{\infty}\left(T \mathbb{R}^{2}\right)$. Therefore,

$$
\nabla_{\widetilde{X}} \widetilde{s}_{0}=\nabla_{\widetilde{X}} \tilde{\phi} \widetilde{t}_{0}=(\widetilde{X} \tilde{\phi}) \widetilde{t}_{0}+\tilde{\phi} \nabla_{\widetilde{X}} \widetilde{t}_{0}
$$

whence we get

$$
\frac{d \tilde{\phi}}{\tilde{\phi}}=\frac{i}{\hbar}\left(\Theta_{\widetilde{P}}-\Theta_{\widetilde{F}}\right)=d\left(\frac{i}{\hbar} \widetilde{\Psi}\right)
$$

(cf. (14)-(15)); thus, $\tilde{\phi}=C e^{i \widetilde{\Psi} / \hbar}$. Similarly for $s_{0}=\phi t_{0}, \phi=C^{\prime} e^{i \Psi / \hbar}$ (cf. (16)). Hence we have

Vol. 33, No. 2, 2015] 
Lemma 2.1. For $t \in \Gamma_{P} L, \widetilde{t} \in \Gamma_{\widetilde{P}} \tilde{L}, s \in \Gamma_{F} L, \widetilde{s} \in \Gamma_{\widetilde{F}} \tilde{L}$, the hermitian products of these polarized sections are given modulo multiplicative constants by the formulas

$$
\begin{aligned}
(\widetilde{t}, \widetilde{s}) & =g(x, y) f\left(z_{-}\left(x, y ; v_{x}, v_{y}\right), \bar{z}_{+}\left(x, y ; v_{x}, v_{y}\right)\right) e^{i \widetilde{\Psi}\left(x, y ; v_{x}, v_{y}\right) / \hbar}, \\
(t, s) & =g\left(x\left(z_{-}, \bar{z}_{-} ; z_{+}, \bar{z}_{+}\right), y\left(z_{-}, \bar{z}_{-} ; z_{+}, \bar{z}_{+}\right)\right) f\left(z_{-}, \bar{z}_{+}\right) e^{i \Psi\left(z_{-}, \bar{z}_{-} ; z_{+}, \bar{z}_{+}\right) / \hbar},
\end{aligned}
$$

with $z_{-}\left(x, y ; v_{x}, v_{y}\right), \bar{z}_{+}\left(x, y ; v_{x}, v_{y}\right)$ and $x\left(z_{-}, \bar{z}_{-} ; z_{+}, \bar{z}_{+}\right), y\left(z_{-}, \bar{z}_{-} ; z_{+}, \bar{z}_{+}\right)$obtained from (9)-(10) and (12), with $\widetilde{\Psi}\left(x, y ; v_{x}, v_{y}\right)$ and $\Psi\left(z_{-}, \bar{z}_{-} ; z_{+}, \bar{z}_{+}\right)$given by (15) and (16).

Remark 2.2. It will be sometimes convenient to rewrite the product $(t, s)$ above as

$$
(t, s)=g\left(\frac{x_{-}+x_{+}}{2}, \frac{y_{-}+y_{+}}{2}\right) f\left(z_{-}, \bar{z}_{+}\right) e^{-\frac{i}{2 \hbar}\left(x_{+} y_{-}-x_{-} y_{+}\right)} e^{-\frac{1}{4 \hbar}\left(\left|z_{-}\right|^{2}+\left|\bar{z}_{+}\right|^{2}\right)} .
$$

Now, as $\widetilde{P}$ is the natural polarization on the tangent bundle, given $q \in \mathbb{R}^{2}$ and $m \in \pi^{-1}(q)$, with $\pi$ the canonical projection, then $T_{q} \mathbb{R}^{2}=T_{m}\left(T \mathbb{R}^{2}\right) / \widetilde{P}_{m}$; thus,

$$
\triangle_{-\frac{1}{2}}\left(\widetilde{P}_{m}\right)=\triangle_{-\frac{1}{2}}\left(T_{m}\left(T \mathbb{R}^{2}\right)\right) \otimes \triangle_{\frac{1}{2}}\left(T_{q} \mathbb{R}^{2}\right),
$$

and so the volume form $\varepsilon$ of $\mathbb{R}^{2}$ determines a $\left(-\frac{1}{2}\right)-\widetilde{P}$-density, given by $\widetilde{v}=\left|\varepsilon_{\Sigma}\right|^{-\frac{1}{2}}|\varepsilon|^{\frac{1}{2}}$, where $\varepsilon_{\Sigma}$ is the volume form of $T\left(T \mathbb{R}^{2}\right)$. Then,

$$
\widetilde{v}\left\{\partial v_{x}, \partial v_{y}\right\}=\left(\left|\varepsilon_{\Sigma}\right|\left\{\partial x, \partial y ; \partial v_{x}, \partial v_{y}\right\}\right)^{-1 / 2} \cdot(|\varepsilon|\{\partial x, \partial y\})^{1 / 2} .
$$

On the other hand, if $J$ is a complex structure compatible with $\Omega$ and the euclidean metric, then $\left(\mathbb{R}^{4}, J\right)$ is a Kähler manifold and the polarization $F$ is a holomorphic polarization; so, there is positive section $v \in \triangle_{-1 / 2}(F)$ uniquely determined by $v \bar{v}=\left|\varepsilon_{\Omega}\right|^{-1 / 2}$, where $\varepsilon_{\Omega}$ is the Liouville volume form in $\mathbb{R}^{2} \times \overline{\mathbb{R}}^{2}$. Hence, we have

$$
v\left\{\partial \bar{z}_{-}, \partial z_{+}\right\}=\left(\left|\varepsilon_{\Omega}\right|\left\{\partial \bar{z}_{-}, \partial z_{+} ; \overline{\partial \bar{z}_{-}}, \overline{\partial z_{+}}\right\}\right)^{-1 / 4} .
$$

Since $\Phi_{*}$ is an isomorphism, for $P=\Phi_{*}(\widetilde{P})$ and $\widetilde{F}=\left(\Phi^{-1}\right)_{*}(F)$, the natural half densites in $\triangle_{-1 / 2}(P)$ and $\triangle_{-1 / 2}(\widetilde{F})$ are given respectively by

$$
v^{\prime}=\left|\left(\Phi^{-1}\right)^{*} \varepsilon_{\Pi}\right|^{-1 / 2}\left|\left(\Phi^{-1}\right)^{*} \varepsilon\right|^{1 / 2}, \quad \widetilde{v}^{\prime}=\left|\varepsilon_{\Phi^{*} \Omega}\right|^{-1 / 4} .
$$

But as $\varepsilon_{\Phi^{*} \Omega}=4\left[d x \wedge d y \wedge d v_{x} \wedge d v_{y}\right]$ (cf. (11)), then we have from (9)-(10) that

$$
\widetilde{v}^{\prime}\left\{\widetilde{\partial \bar{z}_{-}}, \widetilde{\partial z_{+}}, \overline{\overline{\partial \bar{z}_{-}}}, \overline{\widetilde{\partial z_{+}}}\right\}=1 / 2, \quad v^{\prime}\left\{\Phi_{*}\left(\partial v_{x}\right), \Phi_{*}\left(\partial v_{y}\right)\right\}=1 / 2 \sqrt{2},
$$

where $\widetilde{\partial \bar{z}_{-}}=\frac{1}{2}\left(\left(\Phi^{-1}\right)_{*}\left(\partial x_{-}\right)+i\left(\Phi^{-1}\right)_{*}\left(\partial y_{-}\right)\right), \widetilde{\partial z_{+}}=\frac{1}{2}\left(\left(\Phi^{-1}\right)_{*}\left(\partial x_{+}\right)-i\left(\Phi^{-1}\right)_{*}\left(\partial y_{+}\right)\right)$. Therefore, from the definition of the pairing and Lemma 2.1, we have that

$$
\begin{aligned}
& \left\langle t \otimes v, s \otimes v^{\prime}\right\rangle_{p r}= \\
& C \cdot \int_{\mathbb{R}^{4}} g(x, y) f\left(z_{-}, \bar{z}_{+}\right) e^{-\frac{1}{2 \hbar}\left[i\left(x_{+} y_{-}-x_{-} y_{+}\right)\right]} e^{-\frac{1}{4 \hbar}\left(\left|z_{-}\right|^{2}+\left|\bar{z}_{+}\right|^{2}\right)} d x_{-} d y_{-} d x_{+} d y_{+},
\end{aligned}
$$


where $x=\left(x_{+}+x_{-}\right) / 2, y=\left(y_{+}+y_{-}\right) / 2, z_{ \pm}=x_{ \pm}+i y_{ \pm}$and $C$ is a constant.

Now, consider the space

$$
\begin{aligned}
& \mathcal{H} L_{2 \hbar}^{2}(\mathbb{C} \times \overline{\mathbb{C}})= \\
& \left\{f:\left.\mathbb{C}^{2} \rightarrow \mathbb{C}\left|\frac{\partial f}{\partial \bar{z}_{1}}=\frac{\partial f}{\partial z_{2}}=0, \int_{\mathbb{R}^{4}}\right| f\left(z_{1}, \bar{z}_{2}\right)\right|^{2} e^{-\frac{1}{2 \hbar}\left(\left|z_{1}\right|^{2}+\left|z_{2}\right|^{2}\right)} d x_{1} d y_{1} d x_{2} d y_{2}<\infty\right\},
\end{aligned}
$$

where $z_{1}=x_{1}+i y_{1}, z_{2}=x_{2}+i y_{2}$.

Clearly, $\mathcal{H} L_{2 \hbar}^{2}(\mathbb{C} \times \overline{\mathbb{C}}) \simeq \mathcal{H} L_{2 \hbar}^{2}\left(\mathbb{C}^{2}\right)$, the space of holomorphic functions on $\mathbb{C}^{2}$ which are $e^{-|z|^{2} / 2 \hbar} d \mu(z)$-square integrable. Therefore, this is a reproducing kernel Hilbert space (cf. e.g. Theorem 1.4.3(2) in [10]) with reproducing expression given by

$$
f\left(z_{-}, \bar{z}_{+}\right)=\frac{1}{(2 \pi \hbar)^{2}} \int_{\mathbb{R}^{4}} f\left(w_{-}, \bar{w}_{+}\right) e^{\frac{1}{2 \hbar}\left(z_{-} \bar{w}_{-}+\bar{z}_{+} w_{+}-w_{-} \bar{w}_{-}-w_{+} \bar{w}_{+}\right)} d p_{-} d q_{-} d p_{+} d q_{+},
$$

where $w_{-}=p_{-}+i q_{-}$and $w_{+}=p_{+}+i q_{+}$.

Then, with a bit of algebraic manipulation, from (17)-(18) we obtain

$$
\begin{array}{r}
\left\langle t \otimes v, \widetilde{s} \otimes{\widetilde{v^{\prime}}}_{p r}=C^{\prime} \cdot \int_{\mathbb{R}^{8}} \overline{g(x, y)} f\left(w_{-}, \bar{w}_{+}\right) e^{-\frac{i}{\hbar}\left(v_{x} y-x v_{y}\right)} e^{-\frac{1}{2 \hbar}\left(x^{2}+y^{2}+v_{x}^{2}+v_{y}^{2}\right) .}\right. \\
e^{\frac{1}{2 \hbar}\left(x\left(\bar{w}_{-}+w_{+}\right)+i y\left(\bar{w}_{-}-w_{+}\right)+v_{x}\left(w_{+}-\bar{w}_{-}\right)-i v_{y}\left(\bar{w}_{-}+w_{+}\right)\right) .} \\
e^{\frac{1}{2 \hbar}\left(-w_{-} \bar{w}_{-}-w_{+} \bar{w}_{+}\right)} d p_{-} d q_{-} d p_{+} d q_{+} d x d y d v_{x} d v_{y},
\end{array}
$$

and calculating the integrals with respect to $v_{x}$ and $v_{y}$, we get:

$$
\begin{array}{r}
\left\langle t \otimes v, \widetilde{s} \otimes{\widetilde{v^{\prime}}}_{p r}=C^{\prime} \cdot \int_{\mathbb{R}^{6}} \overline{g(x, y)} f\left(w_{-}, \bar{w}_{+}\right) e^{-\frac{1}{\hbar}\left(x^{2}+y^{2}\right)} e^{\frac{1}{\hbar}\left[x\left(\bar{w}_{-}+w_{+}\right)+i y\left(\bar{w}_{-}-w_{+}\right)\right] .}\right. \\
e^{-\frac{1}{2 \hbar}\left(\bar{w}_{-} w_{-}+w_{+} \bar{w}_{+}+\bar{w}_{-} w_{+}\right)} d p_{-} d q_{-} d p_{+} d q_{+} d x d y
\end{array}
$$

whence,

$$
\left\langle t \otimes v, \widetilde{s} \otimes \widetilde{v^{\prime}}\right\rangle_{p r}=\left\langle f, T_{\hbar}[g]\right\rangle_{\mathcal{H} L_{2 \hbar}^{2}(\mathbb{C} \times \overline{\mathbb{C}})}=\left\langle S_{\hbar}[f], g\right\rangle_{L^{2}\left(\mathbb{R}^{2}\right)},
$$

with

$$
S_{\hbar}: \mathcal{H} L_{2 \hbar}^{2}(\mathbb{C} \times \overline{\mathbb{C}}) \rightarrow L^{2}\left(\mathbb{R}^{2}\right)
$$

given by

$$
\begin{array}{r}
S_{\hbar}[f](x, y)=C^{\prime} e^{-\frac{1}{\hbar}\left(x^{2}+y^{2}\right)} \int_{\mathbb{R}^{4}} f\left(w_{1}, \bar{w}_{2}\right) e^{-\frac{1}{2 \hbar} \bar{w}_{1} w_{2}} e^{\frac{1}{\hbar}\left[x\left(\bar{w}_{1}+w_{2}\right)+i y\left(\bar{w}_{1}-w_{2}\right)\right] .} \\
e^{-\frac{1}{2 \hbar}\left(\bar{w}_{1} w_{1}+\bar{w}_{2} w_{2}\right)} d p_{1} d q_{1} d p_{2} d q_{2}
\end{array}
$$

and

$$
T_{\hbar}: L^{2}\left(\mathbb{R}^{2}\right) \rightarrow \mathcal{H} L_{2 \hbar}^{2}(\mathbb{C} \times \overline{\mathbb{C}})
$$

given by

$$
T_{\hbar}[g]\left(w_{1}, \bar{w}_{2}\right)=C^{\prime} e^{-\frac{1}{2 \hbar} w_{1} \bar{w}_{2}} \int_{\mathbb{R}^{2}} g(x, y) e^{-\frac{1}{\hbar}\left(x^{2}+y^{2}\right)} e^{\frac{1}{\hbar}\left[x\left(w_{1}+\bar{w}_{2}\right)-i y\left(w_{1}-\bar{w}_{2}\right)\right]} d x d y,
$$

Vol. 33, No. 2, 2015] 
where we have identified $w_{-}=w_{1}=p_{1}+i q_{1}, w_{+}=w_{2}=p_{2}+i q_{2}$.

Now, in order to pass from $\mathcal{H} L_{2 \hbar}^{2}(\mathbb{C} \times \overline{\mathbb{C}})$ to $\mathcal{H} L_{2 \hbar}^{2}\left(\mathbb{C}^{2}\right)$ and relate to the standard SegalBargmann transform, we conjugate in the second variable, that is, denoting

$$
\mathcal{C}_{2}: \mathbb{C} \times \mathbb{C} \rightarrow \mathbb{C} \times \overline{\mathbb{C}}, \quad\left(w_{1}, w_{2}\right) \mapsto\left(w_{1}, \bar{w}_{2}\right),
$$

we have that, for an appropriate choice of $C^{\prime}$,

$$
T_{\hbar}[g] \circ \mathcal{C}_{2}=\widetilde{\mathfrak{B}}_{\hbar}[g]=\mathfrak{B}\left[g \circ \zeta_{\hbar}^{-1}\right] \circ \eta_{\hbar}: L^{2}\left(\mathbb{R}^{2}\right) \rightarrow \mathcal{H} L^{2}\left(\mathbb{C}^{2}\right)
$$

(cf. (2)-(6) and (22)-(23), where $\mathfrak{B}$ is the standard 2-d Segal-Bargmann transform).

Then, as is well known, the standard Segal-Bargamann transform is an invertible unitary operator $\mathfrak{B}: L^{2}\left(\mathbb{R}^{2}\right) \rightarrow \mathcal{H} L^{2}\left(\mathbb{C}^{2}\right)$ whose inverse can be written as

$$
\mathfrak{B}^{-1}[f](x)=\frac{e^{-\frac{1}{2} x^{2}}}{\pi^{n / 4}} \int_{\mathbb{C}^{2}} f(z) e^{-\frac{1}{2} \bar{z}^{2}+\sqrt{2} x \bar{z}} e^{-|z|^{2}} d \mu(z), \quad x \in \mathbb{R}^{2} .
$$

Therefore, from (24) and (25), using (2)-(6) and (20)-(23) and the discussion following equation (7), we have the following

Theorem 2.3. For an appropriate choice of $C^{\prime}=C_{\hbar}$, the "rotated and scaled" form of the Segal-Bargmann transform $\widetilde{\mathfrak{B}}_{\hbar}$, given by (3) and (24), is an invertible unitary operator $L^{2}\left(\mathbb{R}^{2}\right) \rightarrow \mathcal{H} L_{2 \hbar}^{2}\left(\mathbb{C}^{2}\right)$ whose inverse $\widetilde{\mathfrak{B}}_{\hbar}^{-1}$ can be written as $\widetilde{\mathfrak{B}}_{\hbar}^{-1}[f]=S_{\hbar}\left[f \circ \mathcal{C}_{2}^{-1}\right], S_{\hbar}$ given by (20), from which it follows that $S_{\hbar}: \mathcal{H} L_{2 \hbar}^{2}(\mathbb{C} \times \overline{\mathbb{C}}) \rightarrow L^{2}\left(\mathbb{R}^{2}\right)$ is an invertible unitary operator whose inverse $S_{\hbar}^{-1}=T_{\hbar}$ is given by (22).

\section{Re-deriving the Groenewold-von Neumann product}

Starting from the usual convolution of functions on the symplectic pair groupoid

$$
\left[f_{1} \otimes f_{2}\right]\left(p_{1}, q_{1} ; p_{3}, q_{3}\right)=\int_{\mathbb{R}^{2}} f_{1}\left(p_{1}, q_{1} ; p_{2}, q_{2}\right) f_{2}\left(p_{2}, q_{2} ; p_{3}, q_{3}\right) d p_{2} d q_{2},
$$

and motivated by the measure in the Segal-Bargmann space and the pairing given by equation (19), we define a deformed convolution on $\mathcal{H} L_{2 \hbar}^{2}(\mathbb{C} \times \overline{\mathbb{C}})$ as follows

$$
\begin{aligned}
\otimes_{\hbar}: \mathcal{H} L_{2 \hbar}^{2}(\mathbb{C} \times \overline{\mathbb{C}}) \times \mathcal{H} L_{2 \hbar}^{2}(\mathbb{C} \times \overline{\mathbb{C}}) & \rightarrow \mathcal{H} L_{2 \hbar}^{2}(\mathbb{C} \times \overline{\mathbb{C}}) \\
\left(f_{1}, f_{2}\right) & \mapsto f_{1} \otimes_{\hbar} f_{2},
\end{aligned}
$$

where

$$
\left[f_{1} \otimes_{\hbar} f_{2}\right]\left(w_{1}, \bar{w}_{3}\right)=\int_{\mathbb{R}^{2}} f_{1}\left(w_{1}, \bar{w}_{2}\right) f_{2}\left(w_{2}, \bar{w}_{3}\right) e^{-\frac{1}{2 \hbar} w_{2} \bar{w}_{2}} d p_{2} d q_{2},
$$

which can be straightforwardly checked to satisfy the following

Lemma 3.1. The deformed convolution defined by (26)-(27) above is associative. 
From this, we define a new product on $L^{2}\left(\mathbb{R}^{2}\right)$ as follows

$$
\begin{aligned}
*_{\hbar}: L^{2}\left(\mathbb{R}^{2}\right) \times L^{2}\left(\mathbb{R}^{2}\right) & \rightarrow L^{2}\left(\mathbb{R}^{2}\right) \\
\left(g_{1}, g_{2}\right) & \mapsto g_{1} *_{\hbar} g_{2},
\end{aligned}
$$

where

$$
g_{1} *_{\hbar} g_{2}=S_{\hbar}\left[T_{\hbar}\left[g_{1}\right] \otimes_{\hbar} T_{\hbar}\left[g_{2}\right]\right],
$$

which from Theorem 2.3 and Lemma 3.1 satisfies the following

Corollary 3.2. The product $*_{\hbar}$ defined by (28)-(29) above is associative.

Finally, by a straightforward computation, one can easily check the following

Proposition 3.3. The formula for the product $*_{\hbar}$ defined by (28)-(29) via (20)-(22) and (26)-(27) coincides with formula (8) for the Groenewold-von Neumann product.

As a last remark, we emphasize that the whole treatment presented in this short paper generalizes in an obvious way from $\mathbb{R}^{2}$ to $\mathbb{R}^{2 n}$, for every $n \in \mathbb{N}$.

\section{Acknowledgements}

Heartfelt thanks to Pedro Rios, my thesis advisor, for their critical reading of the manuscript and their many valuable suggestions for improvements.

\section{References}

[1] Bargmann V., "On a Hilbert space of analytis funstions and an associated integral transform", Comm. Pure Appl. Math. 14 (1961), 187-214.

[2] Daubechies I. and Grossmann A., "An Integral transform related to quantization", J. Math. Phys. 21 (1980), No. 8, 2080-2090.

[3] Daubechies I., Grossmann A. and Reignier J., "An Integral transform related to quantization. II. Some mathematical properties", J. Math. Phys. 24 (1983), No. 2, 239-254.

[4] de M. Rios P. and Ozorio de Almeida A., "A variational principle for actions on symmetric symplectic spaces", J. Geom. Phys. 51 (2004), No. 4, 404-441.

[5] Gracia-Bondía J.M. and Várilly J.C., "From geometric quantization to Moyal quantization", J. Math. Phys. 36 (1995), No. 6, 2691-2701.

[6] Folland G.B., Harmonic analysis on phase space, Annals of Mathematics Studies, 122, Princeton University Press, Princeton, NJ, 1989.

[7] Guillemin V. and Sternberg S., Geometric Asymptotics, American Mathematical Society, Providence, RI, 1977.

[8] Hall B.C., "Geometric quantization and the generalized Segal-Bargamann transform for Lie groups of compact type", Comm. Math. Phys. 226 (2002), No. 2, 233-268.

[9] Hall B.C., "Holomorphic methods in analysis and mathematical physics", in First Summer School in Analysis and Mathematical Physics (Cuernavaca Morelos, 1998), Contemp. Math., 260, Amer. Math. Soc., Providence, RI (2000), 1-59.

Vol. 33, No. 2, 2015] 
[10] Moreno J.B., "Construção geométrica de "star product" integral em espaços simpléticos simétricos não compactos" (Portuguese), Thesis (Ph.D), Universidade de São Paulo, São Paulo, 2013, 181 p.

[11] von Neumann J., "Die Eindeutigkeit der Schrodingerschen Operatoren" (German), Math. Ann. 104 (1931), No. 1, 570-578.

[12] Puta M., Hamiltonian mechanical systems and geometric quantization, Kluwer Academic Publishers Group, Dordrecht, 1993.

[13] Rios P. de M. and Tuynman G.M., Weyl quantization from geometric quantization, AIP Conf. Proc., 1079 (2008), 26-38.

[14] Woodhouse N., Geometric quantization, The Clarendon Press, Oxford, 1980. 